\title{
On the revised SI, specifically on the numerical value of the Planck constant
}

\author{
Franco Pavese ${ }^{1}$ \\ ${ }^{1}$ Independent Scientist, Torino, Italy
}

ABSTRACT

The results of the CODATA 2017 adjustment and the consequent stipulation of the numerical values of the constants are essential considerations concerning the consequences of the CGPM accepting the CIPM proposal for the revision of the SI. This paper raises (unresolved) questions on these results, specifically concerning the numerical value of the Planck constant.

\section{Section: RESEARCH PAPER}

Keywords: constants; Planck constant; revised Sl; stipulation; metrology; metre convention

Citation: Franco Pavese, On the revised SI, specifically on the numerical value of the Planck constant, Acta IMEKO, vol. 7, no. 4, article 15, December 2018, identifier: IMEKO-ACTA-07 (2018)-04-15

Editor: Alistair Forbes, National Physical Laboratory, London, UK

Received October 2, 2018; In final form December 4, 2018; Published December 2018

Copyright: @ 2018 IMEKO. This is an open-access article distributed under the terms of the Creative Commons Attribution 3.0 License, which permits unrestricted use, distribution, and reproduction in any medium, provided the original author and source are credited

Corresponding author: Franco Pavese, e-mail: frpavese@gmail.com, frpavese@yahoo.com

\section{INTRODUCTION}

Considering the acceptance of the CGPM of the CIPM proposal for the revision of the SI, this paper analyses the important results of the CODATA (short for CODATA-TGFC) 2017 adjustment reported in [1] (now incorporated in the Draft Resolution A for the $26^{\text {th }}$ CGPM). Study [2] supports the adjustment, providing a deeper analysis than [1] with further details on the method used. In particular, Figure 2 in [2] shows the 2014-2017 data for the Planck constant, $h$, used by CODATA. For convenience, Table 1a reports here the CODATA adjustments since 2006 for $h$, while Table $1 \mathrm{~b}$ of the Appendix reports the same data for the other three constants: $e$, $k$, and $N_{\mathrm{A}}$.

The CCU has basically terminated its task and the CIPM prepared in its 106 2017 Meeting [3] drafts of documents to submit at the $26^{\text {th }}$ meeting of the CGPM in February 2018 Draft Resolution A. Regardless thereof, the intention of this paper is to consider the present information (see $[4,5]$ for an analysis of the previous 2014 drafts) with the aim of making a positive contribution to the scientific debate on some aspects of the revised SI, a debate that certainly shall not stop, as is typical in science. The revision gives rise to some questions that it is the intention of this paper to outline. An Appendix complements some contents of this paper.

\section{ANALYSIS AND COMMENTS}

\section{1. "Exactness" of the stipulated data}

Concerning papers [1] and [2], a reader informed on the SI revision process will note that the number of digits now indicated by the CODATA for the stipulated constants is larger than was previously required, possibly with the exception of $k$. This is certainly due to the lowering of the experimental uncertaintiesan outstanding $\approx 5$ times since 2006 . However, apparently, it is also due to a specific use of the original data and of the associated uncertainties. Table 2 shows both the CODATA adjusted/stipulated numerical values and the "exactly known values."

The CIPM preference expressed in [6] (and that of CCU) has been "for the minimum number of digits [of the stipulated value] for each defining constant $h, e, k$, and $N_{\mathrm{A}}$ of the revised SI that yields consistency factors equal to [exactly] 1 within their uncertainties" (emphasis added). The CODATA stipulations [1,2], presented here in the third column of Table 2, were considered to match this preference, but there are different ways of treating digits with the same numerical values.

Should the "expanded uncertainty", $U$, which is commonly advised to be used in experimental science, be considered for the stipulation of the numerical values? By using $U$, the uncertainties of the numerical values in the second column of Table 2 would be multiplied by $\approx 2$, and the above divergence would not change for any of the four constants if the "exactly known numbers" are 
not rounded. case. When rounded instead, $k$ and $N_{\mathrm{A}}$ are "losing" one further digit.

However, there is another principle, explicit among the CIPM/CCU rules when they speak of "consistency factors," which must also be respected in stipulation: the "continuity principle" [4, 5]. Values of the "consistency factors" can be computed from the stipulated values reported in Table 2 (obtained from the adjusted values in Table 1) and are reported here in the equations below:

$$
\begin{aligned}
& {\left[m(K) /(\mathrm{kg})_{\mathrm{rev}}\right] / 1=1.000000001(10)\left[1.2 \times 10^{-8}\right]} \\
& {\left[\mu_{0} /\left(\mathrm{H} \mathrm{m}^{-1}\right)_{\mathrm{rev}}\right] /\left(4 \pi \times 10^{-7}\right)=1.00000000020(23)\left[2.3 \times 10^{-10}\right]}
\end{aligned}
$$

$\left[M\left({ }^{12} \mathrm{C}\right) /\left(\mathrm{kg} \mathrm{mol}^{-1}\right)_{\mathrm{rev}}\right] / 0.012=1.00000000037(45)\left[4.5 \times 10^{-10}\right]$

$$
\left[T_{\mathrm{TPW}} /(\mathrm{K})_{\mathrm{rev}}\right] / 273.16=1.00000001(37)\left[5.7 \times 10^{-7}\right] .
$$

These factors, basically the same than those now included in CGPM Resolution 1, are considered to correspond with the CIPM's indicated criteria (see the Appendix for their exact meanings). The above equations are the direct consequence of using all CODATA stipulated digits, but they may conflict with the continuity principle (see an example in the Appendix).

The possible conflict is due to the fact that, rather obviously, one should not stipulate a number whose (last) digit(s) are not firmly confirmed by the experiments. Could one stipulated number have, say, three digits more than the experimental uncertainty level? The fact that the uncertainty will eventually be dropped in stipulation is entirely irrelevant: Uncertainty means that the digit(s) affected by it could presently be different from the stipulated one(s).

A basic dilemma arises (apparently undetected, certainly unpublished and unresolved) for the constants in the above situation:

(0) Accepting constant stipulated values exceeding the present experimental accuracy, but preserving unit magnitude continuity

or

(1) Requiring constant stipulated values to conform to present experimental accuracy, but inducing a unit magnitude discontinuity (small but significant).

In fact, the present experimental results, despite their conspicuous five-time uncertainty lowering, still do not support the firm continuity of the units' magnitudes. On the other hand, the CODATA 2017 analysis has been unable to take into account some more recent findings, e.g., on the BIPM Kibble balance [7-9], about possible systematic effects.

To obtain the desired continuity, one is obliged to "guess" the last digit(s) affected by uncertainty (see more in the Appendix).

Furthermore, a formal problem may arise from the fact that the CODATA, a scientific committee, has been required to use, in addition to the LSA adjustment, a second (implicit in [2]) criterion: that of continuity, implying the possible inclusion of stipulated digits affected by uncertainty (see more in the Appendix about that implication and the "adjusting numerical values"). In fact, the CODATA was appointed by the CIPM to make the stipulation proposal, despite that its mission would consist only of providing adjusted values and their uncertainties. However, the issue of continuity is strictly one of the most basic features of the metrology regulatory context and should not be considered a task included within the CODATA's competence.
Confounding the two tasks could be improper in the SI regulatory context.

\subsection{Inconsistency of data and the effect thereof}

Another major issue has arisen since publication of the 2017 CCU document [10] concerning the evident inconsistency of some of the new 2017 data on the Planck constant: "Notes...that work is under way in NMIs to understand the cause for the dispersion of the experimental determinations of the Planck and Avogadro constants..." This was also noted by the CODATA in [1-2]. Nevertheless, the CCU concluded that "numerical values and uncertainties for the Boltzmann constant and the Avogadro constant provided by the CODATA Task Group on Fundamental Constants in their special Least-Squares Adjustment of the experimental data provide a sufficient foundation to support the redefinition..." and recommended that the CIPM should proceed for the $26^{\text {th }}$ CGPM in 2018. The CIPM did so in its 2017 meeting [3].

If data inconsistency is called, as it is by default, evidence of non-overlapping uncertainty intervals (for $k=1$ in the case of the CODATA) for the data, Figure 2 in [2] shows such a case for three portions of the 2017 data, which have been considered as such by CODATA.

However, in [11], the conclusion does not confirm the lack of consistency, as the study is based on a different specific statistical tool. In that paper, musing on the data for the Planck constant (one of the main reasons for the commonly agreed-on urgency of the SI revision), a strong assertion is made: "The preliminary value of the answer to (i) is 10 parts in $10^{9}$, and the discussion in the following sections will only reinforce the view that (ii) should be answered in the affirmative", where the answers were "about (i) the relative uncertainty that the CODATA-TGFC will use to qualify the value recommended for $b$ in the special adjustment of 2017, and about (ii) whether this relative uncertainty warrants the redefinition in the sense that it guarantees seamless continuity in the primary realizations of the unit of mass before and after the redefinition, to within an uncertainty that is comparable to the uncertainties prevailing in the current, routine dissemination of the IPK."

On the contrary, in [12], evidence of inconsistency comes, too, from the use of another analytical method for the same 2017 data on the Planck constant. In this study, the use of a Bayesian method, specifically including the three critical portions of the 2017 data, led to a further conclusion. While the CODATA 2017 adjusted value for $b[1]$ basically results in a value that is equal to that of the NRC-17, from Figures 3-4 in [12] (to be compared with the less clear Figure 2 in [11]), the evidence shows a continuing trend in time towards higher values of $h$ and points rather to the IAC-17 value (which is inconsistent with the CODATA 2017 adjusted value [2]).

That trend in time (see also Table 1a) is still sufficiently significant in 2017 to permit the doubt that the CODATA result and the assertion in [11] are not confirmed by or are insufficiently based on the available data. In fact, in Figures 3-4 of [12], an increase in the credible interval is also shown, another reason for cautiousness about the number of stipulated digits. These facts even led to the conclusion in [12] that "although nothing can be concluded about a possible future development of the CODATA values for the Planck constant, their contingent change over the past decades does not encourage a redefinition of the kilogram at present."

The above are facts that cannot currently be modified until other data would become available. Should no additional 
experimental data be included and analyzed before the final decision, proposing "solutions" is risky: One should not feel sufficiently reassured by either the need to find the "right" statistical test to get a comfortable result [11] or to merely resort to the method of making the uncertainty of such data uninfluential [1, 2].

\subsection{Treatment of inconsistent data}

In [1], it is said (full citation in the Appendix), “...To achieve consistency, multiplicative expansion factors were applied to the uncertainties...The uncertainties of these input data are multiplied by a factor of 1.7. With this expansion of the uncertainties of the eight data, five have relative standard uncertainties $u_{r}$ at or below $50 \times 10^{-9}$, with two at or below $20 \times 10^{-9} \ldots$ " The position is also indicated in [2], where it is specified, "it is note worthy that even after applying an expansion factor of 1.7 to the uncertainties of all...data, thereby bringing them into agreement, the relative uncertainties of the first five values of $h \ldots$ are, in parts in $10^{9}$, only $15,20,23,34$, and 42 , respectively."

The reported "uncertainty lowering" is strictly a feature of the consistency-checking LSA method, a possible weakness in cases like this one. In fact, one should not be particularly surprised by this effect, because it is certainly not the first time that the uncertainty of a constant is reduced thanks to the well-known interconnections that the LSA method establishes between all elements of the dataset. In the present case (a "special adjustment" not involving the usual full set of constants), it might merely indicate that the dispersion of the values for $h$, after having been assigned an uncertainty that is 1.7 times larger, has become almost irrelevant for the general consistency degree of the whole dataset. Here, consistency has a different meaning with respect to the data consistency, discussed in [11, 12] by using specific statistical tools that are different from those of the CODATA. However, since here the uncertainty lowering is not reflected in the experimental findings, it should be considered as an LSA artefact, and the conclusions reported in [12] seem reasonable (see more in the Appendix). Basically, the LSA made the 2017 data uninfluential on the results, i.e., the data and results of the 2017 adjustment for $h$ are in fact the same as the 2014 adjustment, because the same data is basically used.

The LSA method of increasing discrepant data uncertainty to eliminate inconsistencies is common in metrology; however, it should be considered as a better-than-nothing solution, since the discrepancy could be, in reality, not due to an underestimation of the uncertainty but to a real bias of the provided value. In the present case, too, it does have some inconvenience. The fact that the uncertainty remains small after the uncertainty "expansion" is not necessarily good news when the main goal is actually the adjustment of the numerical value. The latter could become impaired by the small sensitivity of the constant's subset with respect to the overall dataset. As a consequence, it may happen that the value is adjusted more or less than is correct (see more in the Appendix).

\section{CONCLUSION: INSUFFICIENCY OF THE PRESENT ANALYSES OF THE 2017 FINAL DATA}

Concerning the 2017 adjusted values in [1], whose stipulations are indicated in $[1,2]$, in general, the presently available analyses should be considered insufficient concerning such an important subject matter. In particular, the CODATA 2017 value of $b$ is not supported by all the presently published analyses. For all new constants, the stipulated values include digits that are not supported by experimental data.

Considering the extraordinary effort made by several NMIs to supply more data in order to support the necessary decisions about the numerical values for assignment to the constants, one would have expected that deeper analyses should have been made available in support of the results of the 2017 CODATA adjustment and, beyond it, to the available dataset, which should ideally be increased.

The importance of the result of the SI revision (not only for metrologists, but for the entire scientific community) should have prompted a broad range of competent and independent analyses, using different methods. In this respect, analyses that are independent of those of CODATA should have been. The advantage would be to mitigate the yet unconsidered effect of the possibly biased values and reduction of uncertainty levels caused by the exclusive use of the LSA consistency check, thus leading to improved evidence concerning the digits needed and allowed for expressing the numerical values of the constantsthe Planck constant in particular. A combined "best value" and its associated uncertainty should be obtained by using several diverse methods (frequentist, Bayesian, etc.), the LSA being one of them. This approach would then lead to higher confidence in the process of stipulation of "exact" numerical values.

A deadline for the start of the validity of the revision farther than the present one, May 20, 2019, is suggested, to fix these issues, and with also their inclusion in the text of the $9^{\text {th }}$ SI Brochure to be finalised by the CIPM.

\section{REFERENCES}

[1] D. B. Newell, F. Cabiati, J. Fischer, K. Fujii, S. G. Karshenboim, H. S. Margolis, E. de Mirandes, P. J. Mohr, F. Nez, K. Pachucki, T. J. Quinn, B. N. Taylor, M. Wang, B. Wood, Z. H. Zhang, The CODATA 2017 values of $h, e, k$, and $N_{\mathrm{A}}$ for the revision of the SI, accepted 20 December 2017, Metrologia 55 (2018) p. 29. See also: CGPM, Resolution 1 of the $26^{\text {th }}$ Meeting, https://www.bipm.org/en/CGPM/db/26 (Access date: 9 January 2019).

[2] P. J. Mohr, D. B. Newell, B. N. Taylor, E. Tiesinga, Data and analysis for the CODATA 2017 special fundamental constants adjustment for the revision of the SI, Metrologia 55 (2018) pp. 125-146.

[3] CIPM, Decisions of the $106^{\text {th }}$ CIPM (October 2017), BIPM, Sèvres [Online] Available: https://www.bipm.org/en/committees/cipm/meeting/ 106.html (Access date: 9 January 2019).

[4] F. Pavese, Are the present measurement standards still valid after SI redefinition? ACQUAL 22 (2017) pp. 291-297.

[5] F. Pavese, The new SI and the CODATA recommended values of the fundamental constants 2014 (arXiv:at-phys 1507.07956), following the CCU 2016 draft of the $9^{\text {th }}$ SI Brochure and its $22^{\text {nd }}$ meeting, a note on the $8^{\text {th }}$ digit for stipulated $\{k\}$, arXiv:physics.data-an, 1512.03668v3, Jun. 13, 2017.

[6] CIPM, Decision CIPM/105-15, 2016 [Online] Available: https://www.bipm.org/en/committees/cipm/meeting/105.html (Access Date: 9 January 2019). See also: CCU Decisions 2016, BIPM, Sèvres, [Online] Available: https://www.bipm.org/cc/ Allowed/22/CCU2016 7july2016 (Access Date: 9 January 2019).

[7] BIPM, Improved understanding of Kibble balance magnets, 2018 [Online] Available: https://www.bipm.org/en/news/fullstories/2018-01-kibble.html (Access Date: 9 January 2019).

[8] S. Li, F. Bielsa, M. Stock, A. Kiss, H. Fang, A permanent magnet system for Kibble balances, Metrologia 54 (2017) pp. 775-783

[9] S. Li, F. Bielsa, M. Stock, A. Kiss, H. Fang, Coil-current effect in Kibble balances: analysis, measurement, and optimization, Metrologia 55 (2018) pp. 75-83. 
[10] CCU, Recommendation U1, On the possible redefinition of the kilogram, ampere, kelvin and mole in 2018, BIPM, Sèvres, 2017 [Online] https://www.bipm.org/cc/AllowedDocuments.jsp?cc=CCU (Access Date: 9 January 2019).

[11] A. Possolo, S. Schlamminger, S. Stoudt, J. R. Pratt, Evaluation of the accuracy, consistency and stability of measurements of the Planck constant used in the redefinition of the international system of units, Metrologia 55 (2018) pp. 29-37.

[12] G. Wübbeler, O. Bodnar, C. Elster, Robust Bayesian linear regression with application to an analysis of the CODATA values for the Planck constant, Metrologia 55 (2018) pp. 20-28.

[13] P. J. Mohr, D. B. Newell, B. N. Taylor, CODATA recommended values of the fundamental physical constants: 2014, arXiv:1507.07956v1, Rev. Modern Physics 88 (2016) pp. 1-73.

[14] E. Tahl, Naturalness and convention in the International System of Units, Measurement 116 (2018) pp. 631-643.

\section{APPENDIX}

\section{On the "exactness" of the stipulated data}

a) Stipulation. Let us compare the CIPM preference, expressed in [6] and shared by the CCU, "for the minimum number of digits for each defining constant $h, e, k$, and $N_{\mathrm{A}}$ of the revised SI that yields consistency factors equal to 1 within their uncertainties" (emphasis added) with the CODATA stipulations [1, 2] in Table 2 of the main text of this paper.

Initially, these uncertainties were required by the CIPM to be those transferred by the constants in Table 1 to the stipulated values of the present SI. Then, they became those representing the state of the art. The reason for the latter preference comes from one of the fundamental principles on which the revised SI is based, i.e., that the numerical stipulated values reported by the present-SI should remain unaltered in the revised-SI when the "continuity principle" is respected.

In addition, let us also remember that "stipulation" is a convention agreed by consensus that rounds up (not truncates) a "best value" (in a statistical sense and at a specific time) into a "reference value" to be then taken as exact, thus characterized by the highest available reliability (confidence level/degree of belief) about its future stability in time. Such a condition implies constraints in choosing the number of stipulated digits, e.g., it requires excluding any of those among the least significant ones that are affected by uncertainty. In fact, the criteria for stipulation cannot be independent on the resulting uncertainty of a chosen value based on experimental evidence, the only available evidence for deciding the number of stipulated (last) digits.

b) In respect of the above CIPM/CCU criterion: an example. According to the Note in [5] concerning the Boltzmann constant, the current reproducibility of a well-operated triple-point cell of water $(\mathrm{TPW})$ is stated to be $(k=1) \approx 10 \mu \mathrm{K}\left(3.7 \times 10^{-8}\right.$ in relative units). Thus, assuming the consistency factor of the new SI with respect to the present one is exactly 1 for the nine-digit CODATA value $1.38064852(79) \times 10^{-23}$ (2014: only a sevendigit exact value), that coefficient should not be lower than 0.999 999 964. By taking an eight-digit CODATA 2014 value, 1.380 $6485 \times 10^{-23}$, one gets 0.99999999 , corresponding to a temperature unit mismatch of $|4| \mu \mathrm{K}$. Meanwhile, taking a seven-digit one (rounded), $1.380649 \times 10^{-23}$, one gets 1.000000 35 , corresponding to a temperature unit mismatch of $|95| \mu \mathrm{K}$. However, one should also consider that the uncertainty provided by CODATA for the nine-digit numerical value corresponds to a temperature uncertainty of $156 \mu \mathrm{K}$ on the $T_{\text {TPW }}$ due to the uncertainty on $k$. This means that the mismatch for the eightdigit format is actually $|4|(156) \mu \mathrm{K}$. In 2017, the nine-digit
CODATA value changed to $1.38064903(51) \times 10^{-23}$, so that, by chance, the eight-digit and seven-digit cases actually coincide.

c) The contrast between the continuity principle and the uncertainty of the experimental outcomes. Should column "exact number" of Table 2 in the main text of this paper be taken as correct in respect of the above considerations, the use of a seventh to tenth $\operatorname{digit}(\mathrm{s})$, depending on the constant, would not be justified, according to the 2017 CODATA expanded uncertainties, $U$, of the experimental outcomes and the 2017 adjustment: $h\left(U \approx 2.2 \times 10^{-8}\right)$, $e\left(U \approx 1.2 \times 10^{-8}\right), N_{\mathrm{A}}\left(U \approx 2.2 \times 10^{-8}\right)$, and $k\left(U \approx 1.5 \times 10^{-6}\right)$.

This would mean that the correct consistency could be realized without invoking any non-exact digit, only for at least one order of magnitude less than is intended and indicated in Equation (1) of the main text of this paper.

d) Inconsistent data and the treatment thereof. The full reference to [1] is: "To achieve consistency, multiplicative expansion factors were applied to the uncertainties of two subsets of input data corresponding to two adjusted constants for the 2017 Special Adjustment. The first subset consists of the eight input data for the Planck and Avogadro constants...relevant to the adjusted value of the Planck constant. The uncertainties of these input data are multiplied by a factor of 1.7. With this expansion of the uncertainties of the eight data, five have relative standard uncertainties $u_{r}$ at or below $50 \times 10^{-9}$, with two at or below $20 \times 10^{-9}$, where the latter includes results from both the Kibble balance and the x-ray crystal density (XRCD) methods." In [2], it is also stated, "it is note worthy that even after applying an expansion factor of 1.7 to the uncertainties of all the Kibble balance and XRCD data, thereby bringing them into agreement, the relative uncertainties of the first five values of $h$ in Table $X$ are, in parts in $10^{9}$, only $15,20,23,34$, and 42 , respectively."

The fact that the uncertainty remains small after the uncertainty "expansion" is not necessarily good news. Problems could possibly be revealed by analyzing the magnitude of the "adjusting numerical values" (ANV), i.e., the values added to the original values in order to obtain the adjusted values. In a normal situation, most of the ANVs are insignificant concerning the uncertainties associated with the adjusted values, but some ANVs may show significant or even anomalously high values. Analysis of the ANVs should be mandatory.

The ANVs were never published by CODATA, although they are necessarily available as an outcome of the LSA method, being the adjustments. Evidence of the difference between the "original" value and the "adjusted" value is reported in [2], where it is indicated: "As in past CODATA adjustments, the uncertainty of a theoretical expression is taken into account by including in the expression an additive correction $\delta$. Each such $\delta$ is taken as an adjusted constant and is included as an input datum with initial value zero but with an uncertainty equal to that of its corresponding theoretical expression." Actually, a variable $\delta$ is associated with each single data piece in the set, with the initial value equal to zero, which is the "adjustment." Its value $\neq$ 0 is then optimized by the LSA procedure. These are ANVs. The CODATA should publish a table of them, in which each outlying datum would appear with a larger $\delta$ with respect to non-outlying data. The existence of an "adjusting numerical value" is disputed concerning the application of the LSA to experimental values of the constants. It is true that in the case of a theoretically computed value in a "law," that value acts as a fixed reference so that the "adjustment" clearly consists of an added $\delta$, computed as in [2]. However, the meaning of "adjustment," a term consistently used in all past and present CODATA studies, 
necessarily implies that there is another numerical value (normally different) for any and each of the "adjusted" quantities in the case that the CODATA LSA method is not applied.

It must be noted that those $\delta$ are the corrections that after stipulation of the numerical value of a constant, the laboratories will not necessarily have to apply to their published inconsistent numerical values in their standards when assessing that their national realization reproduces the stipulated value of the constant. These $\delta$ are the "best" values according to the LSA criteria for obtaining the "adjusted values," which are a dataset that has, for each constant, a certain dispersion from which a representative value for each constant is then chosen. That is what is used for the correction.

In the case of the experimental values of a quantity, the original ones are the published ones, each with an associated uncertainty: a statistical representative value (summary statistics) can be obtained from them, different for each statistical tool chosen, as found in the literature before 2017. None of them are considered an "adjustment" of another one. The LSA method is one of these tools; however, it is not strictly statistical. Its characteristic is that it does not consider the quantities individually; rather, it also uses deterministic relationships between those quantities, linking them with each other with the aim of achieving a more robust evaluation of the quantity numerical values' inter-consistency. That method results in a numerical value for each of the "adjusted" quantities that is usually different from that which is obtained by other means, being influenced by the abovementioned relationships with other quantities of the set, "relationships" here meaning "equations" ("observational equations" in Table 7 [1]) regardless of (or in addition to) the dispersion of the experimental values. In fact, it happened in the past that the resulting CODATA value for some constants changed with respect to the previous "adjustment," even in the absence of any new experimental determination for those constants in the time interval between the two adjustments.

Considering the basic dependence of the LSA results on the observational equations, one might see a possible similarity with some problems concerning "fundamental laws" recently indicated in [14] ${ }^{1}$ : Does the meaning of "adjustment" involve the formulation of these laws, thus also involving their mutual degree of consistency?

\footnotetext{
1 e.g., "the need to maintain simplicity in the expression of theoretical laws constrains the choice of metric conventions for the quantities appearing in these laws...

Law-based metric conventions are specified by reference to one or more scientific laws. Intervals of a quantity are considered equal if and only if their equality is consistent with the specified laws being empirically adequate...

Fundamental theoretical laws, being idealized, do not provide accurate predictions by themselves...

Changes of metric convention may lead to a change in the mathematical form of laws in which affected kinds of quantity appear... Shifting between non-equivalent metric conventions may also affect so-called 'dimensionless' constants...
}

\section{QUESTIONS THAT ARE STILL WITHOUT FIRM ANSWERS}

Q1 CODATA support. How can the CODATA analysis be considered the single criterion for establishing the stipulated values of the four new constants when its value for the Plank constant, at least, is not supported by all the existing analyses [11]?

Q2 Validity in time of the stipulated values. How can the CODATA analysis be considered sufficient, e.g., by [10], when the future trend of the values of some constants cannot yet be considered stable [11] with sufficient confidence/degree of belief, on subsequent add-ons to the results database?

Q3 Data inconsistency. Should a deeper discussion than in [10, 11] on the present inconsistency of some data and on the best provisions for taking the issue into account be made available? If not, why not?

Q4 LSA versus other methods. How can the LSA method be considered the only formal tool for use when the 2017 evidence of data inconsistencies has demonstrated the existing difference between the use of a criterion for only the consistency testing of a given dataset and the use instead of more comprehensive criteria for establishing its precision?

Q5 Stipulated number of digits. Since "stipulation" means a conventional "best value" round-up to a "reference value," then considered exact and fixed for future periods of time, should a stipulated numerical value ensure the future stability of all its digits at the highest possible confidence level/degree of belief?

Q6 Use of expanded uncertainty. Considering the importance of the result of the SI revision, why is the use of expanded uncertainty (which is common practice in science for important issues) not considered mandatory for determining the stipulated values?

Q7 Unit-magnitude continuity. For expanded uncertainties of experimental outcomes, should the fact that the consistency degree is at least one order of magnitude worse than aimed (thus possibly inducing a critical non-continuity degree for some of the unit magnitudes) require a deep, open discussion about possible provisions?

Q8 Meaning of "adjustment." Since “adjusted" implies the existence of another (normally different) numerical value in the case that the LSA method is not applied, what is the reason why this term is used, if such "original" value cannot be identified? Otherwise, how does its need arise from the original value or from the theoretical relationships between constants, and which $\delta$ is explicitly that resulting from the application of the LSA for each of the constants in the set?
... by fixing the numerical value of a constant, one assumes that it is indeed constant. For example, in fixing the numerical value of the Planck constant, one presupposes that the energy and frequency of a photon have a constant ratio. After all, constants are relations among quantities in scientific laws. Postulating their constancy necessarily involves the assumption that some (or all) of the fundamental laws in which those constants feature are empirically adequate...

Proportionality assumption: the correct way of measuring the quantities of physics is such that the currently known fundamental laws of physics preserve their mathematical form...

The direct incorporation of elements from fundamental theory into the definitions of measurement units carries with it clear advantage. If the above analysis is correct, this incorporation also generates an implicit tension between the need for long-lasting unit definitions and the need for testable fundamental laws." 
Table 1a. Change in numerical value and its uncertainty $u$ of the CODATA adjustments 2006-2017, for the new constant $k$ in the revised SI definition. For $e, k$, and $N_{A}$ in Table $1 b$, see the Appendix.

\begin{tabular}{|c|c|c|c|c|c|}
\hline Constant & CODATA & Numerical value $*$ & $u /$ relative $\times 10^{7}$ & Change & Total shift \\
\hline \multirow{4}{*}{$\begin{array}{l}\text { Planck } \\
b \times 10^{34}\end{array}$} & 2006 & $6.626069_{0}$ & $3.3 / 0.50$ & - & \\
\hline & 2010 & 6.6260696 & $2.9 / 0.44$ & $6 \times 10^{-7}$ & \\
\hline & 2014 & $6.6260700_{4}^{a}$ & $0.8 / 0.12$ & $4.4 \times 10^{-7}$ & $10 \cdot 4 \times 10^{-7}$ \\
\hline & 2017 & $6.62607015^{a}$ & $0.7 / 0.11$ & $1.1 \times 10^{-7}$ & $11.5 \times 10^{-7}$ \\
\hline
\end{tabular}

* The CODATA reports standard uncertainty $u$. The smaller case digits, obtained from the CODATA two-digit uncertainty format, are those affected by uncertainty.

a The 2014 CODATA outcome is $6.626070040(81)$; therefore, the numerical value can be as low as 6.626069959 , thus also affecting the preceding digit. Similarly, for the 2017 one, the CODATA outcome is $6.626070150(69)$. Consequently, the upper bound of the 2017 interval is 6.626070121 , and the lower bound of the 2017 interval is 6.626070081 , not significantly overlapping.

Table $1 \mathrm{~b}$. Change in numerical value and its uncertainty $u$ of the CODATA adjustments 2006-2017, for the new constants $e, N_{\mathrm{A}}$, and $k$ involved in the revised $\mathrm{SI}$ definition. See Table 1 a for $h$ in the main text.

\begin{tabular}{|c|c|c|c|c|c|}
\hline Constant & CODATA & Numerical value $*$ & $u /$ relative $\times 10^{7}$ & Change & Total shift \\
\hline Electron charge & 2006 & $1.6021764_{9}$ & $0.4 / 0.23$ & - & \\
\hline \multirow[t]{3}{*}{$e \times 10^{19}$} & 2010 & $1.6021765_{7}$ & $0.35 / 0.19$ & $8 \times 10^{-8}$ & \\
\hline & 2014 & $1.6021766_{2}$ & $0.1 / 0.06$ & $5 \times 10^{-8}$ & $1.3 \times 10^{-7}$ \\
\hline & 2017 & $1.60217663_{4}{ }^{b}$ & $0.08 / 0.05$ & $1.4 \times 10^{-8}$ & $1.4_{4} \times 10^{-7}$ \\
\hline Avogadro & 2006 & $6.022141_{8}$ & $3.0 / 0.50$ & - & \\
\hline \multirow[t]{3}{*}{$N_{\mathrm{A}} \times 10^{-23}$} & 2010 & $6.022141_{3}$ & $2.7 / 0.45$ & $-5 \times 10^{-7}$ & \\
\hline & 2014 & $6.0221408^{c}$ & $0.7 / 0.12$ & $-4 \times 10^{-7}$ & $-9 \times 10^{-7}$ \\
\hline & 2017 & $6.0221407_{6}^{c}$ & $0.6 / 0.10$ & $1 \times 10^{-7}$ & $-8 \times 10^{-7}$ \\
\hline Boltzmann & 2006 & $1.38065_{04}{ }^{\mathrm{d}}$ & $24 / 17$ & - & \\
\hline \multirow[t]{3}{*}{$k \times 10^{23}$} & 2010 & $1.38064_{88}{ }^{\mathrm{d}}$ & $13 / 9.4$ & $-1.6 \times 10^{-6}$ & \\
\hline & 2014 & 1.3806485 & $8 / 8.5$ & $-0.3 \times 10^{-6}$ & $-1.9 \times 10^{-6}$ \\
\hline & 2017 & $1.380649^{\mathrm{e}}$ & $5 / 3.6$ & $-0.5 \times 10^{-6}$ & $-1.4 \times 10^{-6}$ \\
\hline
\end{tabular}

* The CODATA reports standard uncertainty $u$. The smaller case digits are uncertain, taken from the CODATA two-digit uncertainty format-except for Boltzmann constant $k$, see notes c) and e).

b The 2017 CODATA outcome is 1.602176 6341(83); therefore, the numerical value can be as low as 1.6021766258 and as high as 1.602176 6424, thus also affecting the preceding digit.

c The 2014 CODATA outcome [13] is 6.022140 857(74); therefore, the numerical value can be between 6.022140783 and 6.022140 931, thus affecting the preceding digit. The analysis is similar for the 2017 one, where the CODATA outcome is 6.022 140 758(62).

$\mathrm{d}$ Two digits are shown because the rounding affects also the preceding digit.

e The CODATA 2017 outcome is 1.380649 03(51); thus, the rounding does not include uncertain digits (the only occurrence in the Table). However, the numerical value can be as low as 1.38064850 , thus in fact affecting the last digit.

Table 2. Different ways of treating the digits with the same numerical values for the four constants.

\begin{tabular}{cccc}
\hline Constant numerical value & CODATA 2017 $(k=1)[1]$ & Stipulated ${ }^{\mathrm{a}}[1]$ & Exactly known number ${ }^{\mathrm{b}}$ \\
\hline$\{b\} \times 10^{34}$ & $6.626070150(69), u_{\mathrm{rel}}=1.1 \times 10^{-8}$ & $6.62607015, u_{\mathrm{rel}}=7.5 \times 10^{-10}$ & $6.626070(1), u_{\mathrm{rel}}=2.3 \times 10^{-8}$ \\
\hline$\{e\} \times 10^{19}$ & $1.6021766341(93), u_{\mathrm{rel}}=0.6 \times 10^{-8}$ & $1.602176634, u_{\mathrm{rel}}=3.1 \times 10^{-10}$ & $1.6021766(3), u_{\mathrm{rel}}=4.0 \times 10^{-7}$ \\
$\left\{N_{\mathrm{A}}\right\} \times 10^{-23}$ & $6.022140758(62), u_{\mathrm{rel}}=1.1 \times 10^{-8}$ & $6.02214076, u_{\mathrm{rel}}=8.3 \times 10^{-10}$ & $6.022140(8), u_{\mathrm{rel}}=1.3 \times 10^{-7}$ \\
$\{k\} \times 10^{23}$ & $1.38064903(51), u_{\mathrm{rel}}=7.4 \times 10^{-7}$ & $1.380649, u_{\mathrm{rel}}=3.6 \times 10^{-7}$ & $1.3806(5), u_{\mathrm{rel}}=3.5 \times 10^{-5}$ \\
\hline
\end{tabular}

a The smaller case digits in the proposed stipulations are used here for those affected by the uncertainty interval in the previous column.

b Here "exact" means unaffected by the original experimental uncertainty. The added smaller case digit in parentheses is not exact, being affected by the CODATA uncertainty interval indicated in the previous column: It is reported because the rounding up, different from truncation, would affect the exact numerical value of $N_{\mathrm{A}}$ and $k$. 